\section{Histoire Épistémologie Langage}

43-1 | 2021

La grammaire grecque étendue

\title{
L'« hypothèse grecque » et le débat sur les origines de la tradition grammaticale arabe
}

Jean-Patrick Guillaume

\section{OpenEdition}

\section{Journals}

Édition électronique

URL : https://journals.openedition.org/hel/635

DOI : $10.4000 /$ hel.635

ISSN : 1638-1580

Éditeur

Société d'histoire et d'épistémologie des sciences du langage

Édition imprimée

Date de publication : 30 juin 2021

Pagination : 61-72

ISBN : 9791091587143

ISSN : 0750-8069

Référence électronique

Jean-Patrick Guillaume, «L'« hypothèse grecque » et le débat sur les origines de la tradition grammaticale arabe », Histoire Épistémologie Langage [En ligne], 43-1 | 2021, mis en ligne le 28 octobre 2021, consulté le 29 octobre 2021. URL : http://journals.openedition.org/hel/635 ; DOI : https:// doi.org/10.4000/hel.635

\section{(c) (i) $(9)$}

HEL is licensed under a Creative Commons Attribution-NonCommercial-NoDerivatives 4.0 International License 


\section{L'《 HYPOTHÈSE GRECQUE » \\ ET LE DÉBAT SUR LES ORIGINES \\ DE LA TRADITION GRAMMATICALE ARABE}

\section{Jean-Patrick Guillaume}

Université Sorbonne Nouvelle, Histoire des théories linguistiques (UMR 7597, HTL), Paris, France

\begin{abstract}
Résumé - La question de l'influence grecque sur les origines de la tradition grammaticale arabe fait l'objet d'un débat récurrent depuis la fin du XIX ${ }^{\mathrm{e}}$ siècle. Après en avoir précisé les termes, cet article s'attache à résumer les positions en présence et à en examiner les principaux arguments, puis à illustrer les difficultés soulevées par l'identification des emprunts possibles de l'arabe au grec. La conclusion qui s'en dégage est que, si l' " hypothèse grecque » fournit un scénario plausible des origines de la grammaire arabe, elle reste dans l'état actuel des connaissances impossible à valider avec certitude.
\end{abstract}

Mots-clés - Carter M., diábasis, écoles de Bașra et de Kūfa, influence grecque sur la tradition grammaticale arabe, Merx A., ta'addī, Talmon R., tradition grammaticale arabe (origines), transitivité, Versteegh $\mathrm{K}$.
Abstract - The question of the Greek influence on the origins of the Arabic grammatical tradition has been a controversial subject since the late 19th century. After having clarified the terms of the debate, this paper gives an overview of the positions in presence together with their respective arguments, then proceeds to illustrate the problems raised by the identification of possible Greek borrowings in Arabic. The conclusion which emerges is that, while the "Greek theory" provides us with a plausible storyboard about the origins of Arabic grammar, it remains impossible to prove with certainty in the present state of knowledge.

Keywords - Arabic grammatical tradition (origins), Bașran and Kūfan schools, Carter M., diábasis, Greek influence on the Arabic grammatical tradition, Merx A., ta'addī, Talmon R., transitivity, Versteegh K.

\section{PRÉLIMINAIRES}

Bien que la tradition grammaticale arabe ne relève pas à proprement parler de la grammaire grecque étendue, il n'est sans doute pas hors de propos, dans le cadre de ce dossier, d'évoquer la question des influences que la tradition linguistique grecque a pu exercer sur ses premiers développements, et le débat récurrent qui oppose les partisans de ce que l'on nomme parfois l'" hypothèse grecque » aux défenseurs d'une genèse purement autochtone. Ce débat dure depuis quelque cent trente ans : autant dire que je n'ai pas l'ambition de le trancher - à supposer que la chose soit possible -, ni même d'y apporter grand-chose de nouveau, et que je me bornerai à en préciser les enjeux, à montrer, à partir de quelques exemples, 
pourquoi il n'est jamais parvenu à une solution qui fasse consensus et à tenter d'évaluer ce qu'il a apporté à l'histoire de la tradition linguistique arabe.

Avant d'entrer en matière, il est nécessaire, pour l'intelligence de ce qui suit, de préciser un point important : le débat en question concerne essentiellement la période de formation de la grammaire arabe, c'est-à-dire en gros les $\mathrm{VIII}^{\mathrm{e}}$ et $\mathrm{IX}^{\mathrm{e}}$ siècles. À partir du $\mathrm{X}^{\mathrm{e}}$ siècle, dans un contexte marqué par la diffusion dans les milieux savants d'une culture philosophique hellénisante, la falsafa, l'existence d'emprunts à la tradition grecque - et principalement à la logique - est amplement documentée et ne fait guère de doute. On peut en citer quelques-uns à titre d'exemple :

- deux outils méthodologiques très généraux, la définition par genre prochain (jins) et différence spécifique ( $f a r q$ ), et le principe des divisions exhaustives (alhașr wa-l-taqsīm $)^{1}$ : ils sont mis à l'œuvre pour la première fois dans le Kitāb aluṣūl d'Ibn al-Sarrāj (mort en 928) ;

- la définition canonique des trois parties du discours, nom, verbe et particule (voir Guillaume 2019) ${ }^{2}$;

- la théorie de la prédication sous sa forme canonique, distinguant un noyau prédicatif constitué d'un «support» (musnad ilay-hi) et d'un « apport» (musnad), auquel s'ajoutent des « compléments » (fadalāt : voir Guillaume 2017) ${ }^{3}$;

- la définition du mot (kalima) en termes d'unité signifiante minimale, chez Zamahšarī (mort en 1144) et ses continuateurs (voir Guillaume 2011).

Pour la période précédente, en revanche, les choses se compliquent. Cela tient, pour une grande part, à la rareté des données (notamment historiographiques et prosopographiques) dont nous disposons et aux problèmes d'interprétation qu'elles posent. Quelques points, cependant, peuvent être considérés comme établis, qu'il faut rappeler brièvement ${ }^{4}$. Les premières traces d'une réflexion grammaticale apparaissent dans le courant du VIII ${ }^{\mathrm{e}}$ siècle, principalement dans le milieu des « lecteurs » (qurra $\bar{a})$, spécialistes du texte coranique, dont ils s'emploient à fixer le texte en vue de sa récitation ${ }^{5}$ (Blachère 1952-1966 : I, 108-110). Avec le Kitāb de Sībawayhi (mort en 796 ?) apparaît toutefois un changement de perspective : c'est désormais la langue elle-même qui fait l'objet d'une description systématique et détaillée. Cet ouvrage monumental demeurera néanmoins seul en

1 Opération consistant à diviser un genre (e.g. le mot) en espèces (e.g. nom, verbe et particule), puis chaque espèce en sous-espèces (e.g. le nom déclinable et le nom indéclinable) et ainsi de suite (le nom déclinable se divise en nominatif, accusatif et génitif; le nominatif peut être sujet d'une phrase verbale, thème ou propos d'une phrase thématique etc.). Ce procédé permet en principe un classement systématique et exhaustif de la matière grammaticale (les exemples sont tirés du Kitāb al-Uṣūl; voir la notice CTLF : http://ctlf.ens-lyon.fr/n_fiche.asp?n=345).

2 Précisons bien qu'il s'agit uniquement de leur définition : le système lui-même est en place dès le Kitāb de Sībawayhi.

3 Ici encore, il faut souligner que le terme musnad est déjà présent chez Sībawayhi ; toutefois il semble difficile d'affirmer qu'il est intégré à une théorie générale de la prédication.

4 On trouvera une présentation plus détaillée dans Baalbaki 2008 : 1-30.

5 Ce sont les homologues des massorètes de la tradition hébraïque et des maqreyyane de la tradition syriaque (King 2012). 
son genre jusqu'à la fin du $\mathrm{IX}^{\mathrm{e}}$ siècle, quand, grâce notamment à l'action d'alMubarrad, il s'imposera comme le texte fondateur et la référence incontournable de la grammaire (Humbert 1995 ; Bernards 1997).

Les grammairiens antérieurs à ou contemporains de Sībawayhi ont fait l'objet de jugements très divergents. Le débat s'est particulièrement focalisé sur un groupe mentionné une vingtaine de fois dans le Kitāb, presque toujours de manière critique, sous la dénomination al-nahwiyyūn ${ }^{6}$. Selon Mike Carter (1985; 2004 : 4-6), il s'agirait tout au plus de " proto-grammairiens », dont les analyses encore rudimentaires et primitives ne sauraient se comparer au système pleinement élaboré de Sībawayhi, en qui il voit le véritable fondateur de la discipline $^{7}$. Cette interprétation a été contestée par Rafael Talmon (1982, 2000 et 2003 : passim) : pour lui, les nahwwiyyūn sont les représentants d'une authentique école grammaticale, qu'il nomme la « vieille école irakienne » (Old Iraqi School), dont la doctrine aurait été en partie reprise et en partie remplacée par celle de Sībawayhi et de son maître al-Halīl (mort en 791 ?), qui apparaîtraient non pas comme les fondateurs d'une tradition, mais comme les réformateurs d'une doctrine constituée antérieurement; celle-ci se serait toutefois maintenue un peu plus longtemps dans l'école de Kūfa, rivale de celle de Bașra à laquelle appartiennent les deux précités. Toutefois, en dépit du travail de dépouillement considérable mené par Talmon, le caractère fragmentaire et souvent contradictoire des données existantes ne permet pas de reconstituer véritablement la doctrine de cette « vieille école irakienne », ni même de décider si elle avait atteint un degré d'homogénéité et de complétude comparable à celle du Kitāb. Comme le souligne Ramzi Baalbaki ${ }^{8}$ :

The scarce and inconsistent nature of the information provided by the extant sources of the second/eighth and third/ninth centuries is thus hardly supportive of any theory which tries to explain with reasonable precision either the theoretical bases of the differences which exist among the grammarians who allegedly belong to a tradition which is not identical with Sïbawayhi's, or the so-called "innovations" which Halīl and Sībawayhi are thought to have introduced to grammatical theory. (Baalbaki $2008: 21$ )

\section{L'HYPOTHÈSE GRECQUE ET SES CRITIQUES}

Prise en elle-même, l'hypothèse grecque repose sur un constat qui relève de l'évidence : la grammaire arabe est née dans un contexte qui appartient encore, par de nombreux traits, à l'Antiquité tardive. Il est bien connu, en particulier, que les textes fondamentaux de la tradition linguistique grecque (la Technè de Denys le Thrace, l'Organon et ses commentaires) ont largement circulé dans tout le Proche-Orient byzantin et sassanide, avant et après les conquêtes islamiques, en

6 Dans l'usage établi, il se traduirait par «les grammairiens »; mais c'est précisément son interprétation chez Sỉbawayhi qui pose problème

7 "Even if the first interest in grammar dates from a century earlier, grammar as a science only emerged in Sïbawayhi's lifetime” (Carter $2004: 4)$

8 Voir également Baalbaki 2005. 
grec, mais surtout en syriaque, et parfois en pehlvi (Teixidor 2003 ; HugonnardRoche 2013). Nous savons aussi qu'ils ont été assez tôt accessibles en arabe : une première version, très libre, des quatre premiers livres de l'Organon a été donnée, sans doute d'après un intermédiaire pehlvi, par ${ }^{\complement} A$ bdallāh Ibn al-Muqaffa ${ }^{\varsigma}$ (mort en 756) ${ }^{9}$ ou par son fils Muḥammad (mort vers 815) ; de même, Ḥunayn ibn Isḥāq (mort en 873), l'un des principaux traducteurs en arabe du corpus philosophicoscientifique grec, aurait composé une grammaire arabe " à la façon des Grecs » (Kitāb aḥkām al-i'rāa b 'alā maḍāhib al-Yunāniyyīn: Ibn al-Nadīm, Fihrist, I, 294). L'ouvrage a disparu ${ }^{10}$, mais il s'agissait probablement d'une adaptation à l'arabe de la Technè, destinée aux traducteurs ${ }^{11}$. Bref, il existait de très nombreuses occasions de contact entre la grammaire arabe naissante et la tradition linguistique gréco-syriaque déjà établie, et il paraît à première vue naturel de supposer que la première a pu à tout le moins emprunter quelques outils à la seconde. C'est toutefois lorsque l'on cherche à identifier précisément ces outils que les difficultés apparaissent : le simple fait que la question fasse débat depuis quelque cent trente ans suffit à le démontrer. En témoignent aussi les différentes variantes que l'hypothèse grecque a connues au cours de cette période.

La plus ancienne est celle du sémitisant Adalbert Merx (1838-1909), qui apparaît dans une sorte de développement annexe à l'intérieur d'un ouvrage consacré à l'histoire de la grammaire syriaque (Merx 1889 : 137-154). Selon lui, c'est à la logique aristotélicienne que les grammairiens arabes auraient emprunté leurs notions de base. Cette affirmation passablement improbable ${ }^{12} \mathrm{~s}^{\prime}$ appuie sur un raisonnement qui peut se résumer ainsi :

- La grammaire arabe ne peut pas être le résultat d'un développement autochtone, car il est inconcevable que les Arabes aient produit une grammaire en un siècle, alors qu'il en a fallu plus de deux aux Grecs (de Platon aux Stoïciens) pour parvenir au même résultat.

- En conséquence, ils ont nécessairement emprunté les notions de base de leur grammaire à une source étrangère, qui ne pouvait être que la grammaire syriaque ${ }^{13}$ ou la logique aristotélicienne.

- La première hypothèse, qui serait a priori la plus probable, est néanmoins exclue, puisque les grammairiens arabes ne reconnaissent que trois parties du

9 D'origine persane et zoroastrien converti à l'islam, il est surtout connu pour son adaptation en arabe du Livre de Kalīla et Dimna, un « miroir des princes » sous forme de fables animalières, connu dans l'Occident médiéval sous le nom de Fables de Pilpay.

10 Il semble toutefois que quelques fragments en aient subsisté dans une version judéo-arabe : voir Vidro 2020. Je remercie le relecteur anonyme qui m'a fourni cette information.

11 Pour une exposition plus détaillée de ce qui précède, voir Versteegh 1977 : 1-5. Sur les relations possibles entre la tradition logico-grammaticale syriaque et la grammaire arabe, voir également King 2012 et 2013 .

12 Il suffit d'avoir lu deux pages du Kitāb pour conclure avec Gérard Troupeau que « l'influence de la logique aristotélicienne sur la terminologie grammaticale de Sībawayhi paraît devoir être pratiquement inexistante » (Troupeau $1976: 13$ ).

13 C'est-à-dire, comme chacun sait, l'adaptation au syriaque de la Technè de Denys le Thrace. Notons cependant - ce que relève également Merx - que la terminologie arabe des marques vocaliques est empruntée à la tradition syriaque (voir King 2012). 
discours (nom, verbe et particule), alors que la grammaire syriaque en compte $\mathrm{sept}^{14}$. C'est donc la logique aristotélicienne qui leur a servi de source.

Merx donne ensuite une liste de six emprunts présumés (ce qui, soit dit en passant, semble peu pour produire toute une grammaire); le seul qui garde quelque plausibilité aujourd'hui concerne précisément le système des parties du discours, les autres étant triviaux ou réfutés (Elamrani-Jamal 1983 : 24-35). Par ailleurs, la thèse de Merx, à l'époque où elle a été produite, se heurtait à un obstacle chronologique, dans la mesure où les premières traductions de l'Organon en arabe, pensait-on, dataient du début $\mathrm{du} \mathrm{X}^{\mathrm{e}}$ siècle, soit bien après la période de constitution de la grammaire arabe. Toutefois, la redécouverte et la publication de la vieille traduction d'Ibn al-Muqaffa ${ }^{\varsigma}$ a pu donner quelque espoir aux tenants de la thèse aristotélicienne (Rundgren 1976) ; il n'en reste pas moins que, comme l'a montré Gérard Troupeau, la terminologie d'Ibn al-Muqaffa ${ }^{\varsigma}$, si elle diffère fortement de celle des traductions postérieures, n'a pas davantage de rapport avec celle de Sĩbawayhi ${ }^{15}$ (Troupeau 1981).

Kees Versteegh $(1977 ; 1993$ : 22-28) propose quant à lui une version différente de l'hypothèse grecque, en distinguant une influence directe qui se serait exercée sur la grammaire à partir des $\mathrm{IX}^{\mathrm{e}}-\mathrm{X}^{\mathrm{e}}$ siècles, par le biais de traductions de textes logico-philosophiques grecs, et une influence indirecte, à la période précédente, par ce qu'il nomme "voie diffuse " (en français dans le texte), autrement dit par des contacts informels avec le système éducatif grécobyzantin :

According to this view, the Arabs became acquainted with Hellenistic culture and scholarship in a watered down version as it was taught in the schools all over the Byzantine Empire. In this way, they were able to borrow some of the elements of Greek grammatical teaching without thereby taking over the entire system. As in other aspects of Greek-Byzantine culture (e.g. law and theology), this influence took the form of a diffusion of isolated elements from the provincial cultural atmosphere. (Versteegh $1993: 25$ )

Cette hypothèse apparaît à première vue nettement plus plausible que la précédente : reposant sur l'idée d'une continuité entre l'Antiquité tardive et la première période islamique, elle évite néanmoins de donner à l'influence grecque supposée une importance qu'elle n'a pas, et la limite à l'emprunt d'éléments isolés, sans prétendre offrir une explication globale des origines de la grammaire arabe. Elle a toutefois l'inconvénient d'être à peu près impossible à démontrer ${ }^{16}$ : comme l'a fait remarquer Abdelali Elamrani-Jamal, la «voie diffuse » est une notion trop peu contrainte pour permettre de distinguer d'éventuels emprunts de ressemblances fortuites, ou de convergences dues à des évolutions parallèles (Elamrani-Jamal 1983 et 1984). Cela étant, Kees Versteegh - ainsi que d'autres travaillant dans la même perspective - a proposé un certain nombre de rapprochements qui paraissent plausibles. L'un des plus intéressants est 
probablement le fait que les deux traditions ont recours aux mêmes unités lexicales comme exemples typiques du nom et du verbe, «homme» (rajul, anthrōpos) et «cheval» (faras, híppos) pour le premier, «frapper» (daraba, tuptō) pour le second ${ }^{17}$. On peut en dire autant, à première vue, de l'arabe $t a^{\varsigma} a d d \bar{\imath}$ et du grec metabasis : les deux termes désignent le même objet linguistique (la transitivité) au moyen de la même métaphore (l'idée de traverser, de franchir une limite). D'autres en revanche semblent plus sujets à caution, par exemple le rapprochement entre harf et stoicheion: l'un et l'autre désignent sans doute à peu près le même objet (la lettre et le son qu'elle représente), mais il est peu probable que les Arabes, qui pratiquaient l'écriture bien avant la grammatisation, n'aient pas eu de terme pour désigner les lettres de l'alphabet au point de devoir l'emprunter aux Grecs. On peut dire la même chose de kalām et logos : l'un et l'autre peuvent sans doute désigner techniquement l'énoncé, mais les deux termes, qui appartiennent au fonds ancien de la langue, antérieur à la grammatisation, reflètent des conceptions du langage qui n'ont pas grand-chose en commun (voir Ildefonse \& Lallot 2017 ; Guillaume 2017).

Dans une perspective un peu différente, Rafael Talmon a suggéré dans plusieurs travaux (notamment Talmon 1990, 1993 et 2005) que la doctrine de la « vieille école irakienne » (voir supra) pourrait être en grande partie inspirée de la tradition logico-grammaticale gréco-syriaque, par le biais de petits manuels de logique du genre de celui d'Ibn al-Muqaffa ${ }^{\varsigma}$; cet héritage se serait ensuite perdu dans la doctrine « réformée » de Halīl et Sībawayhi, ou n'aurait subsisté que sous forme de traces imperceptibles. L'hypothèse est évidemment séduisante et permet de proposer un scénario éminemment plausible et cohérent pour expliquer la genèse de la grammaire arabe en la rattachant fermement au contexte de l'Antiquité tardive, sans pour autant sous-évaluer son caractère profondément original. Cela étant, elle ne peut s'appuyer - de l'aveu même de son auteur - que sur de rares indices, extrêmement ténus et difficiles à interpréter de surcroît. Il s'agit donc tout au plus d'une piste, que la disparition prématurée de Rafael Talmon en 2004 ne lui a sans doute pas permis de poursuivre jusqu'à son terme.

On ne saurait quitter ce passage en revue sans mentionner l'adversaire le plus résolu et le plus constant de l'hypothèse grecque, à savoir Mike Carter. Depuis sa thèse de doctorat, soutenue en 1968 et récemment publiée avec de nombreux addendas (Carter 2016 ; voir aussi Carter 1972 et 1973), il a toujours soutenu que la grammaire en tant que discipline autonome commence avec le Kitāb de Sībawayhi et que celui-ci a trouvé sa principale source d'inspiration dans le droit musulman (fiqh) dont il a appliqué les méthodes à l'étude du comportement linguistique des Arabes. Cette thèse a assurément des points forts : il est incontestable que les deux disciplines ont rapport à la norme et que, dans le contexte de l'Islam classique et médiéval, elles se sont développées en étroite symbiose : de nombreux grammairiens, comme Abū l-Barakāt al-'Anbārī (mort en 1181) ou Ibn al-Hāgăib (mort en 1245) étaient aussi d'éminents juristes. Il n'en reste pas moins qu'à l'époque de Sībawayhi, il est douteux que le droit ait atteint un degré 
d'élaboration théorique tel que son modèle fût exportable dans le domaine de la grammaire : le plus ancien document dont nous disposions en la matière, l'Épître sur les fondements du droit (Risāla fì ușūl al-fiqh) d'al-Šāfi'ī (mort en 820) est postérieur au Kitāb et suggère plutôt le contraire.

Cela étant, on admettra volontiers avec Mike Carter que Sỉbawayhi est certainement le premier à avoir conçu et mené à bien le projet d'une description systématique et détaillée de la langue, et qu'il peut, en ce sens, être considéré comme le fondateur de la grammaire comme domaine de savoir autonome et non plus comme une branche auxiliaire des études coraniques, ou comme une discipline purement utilitaire, destinée à inculquer aux néo-arabisés les règles élémentaires du marquage casue $1^{18}$. On admettra tout autant que la grammaire arabe, depuis Sïbawayhi et pendant toute son histoire, présente un air de famille marqué avec le droit musulman. Mais, dans le même temps, on ne peut manquer de lui trouver aussi un air de famille tout aussi marqué avec la tradition linguistique grecque. Toutefois, quand on cherche à dépasser cette impression globale et à lui donner un contenu précis, les difficultés apparaissent. C'est ce que l'on voudrait illustrer par un exemple concret.

\section{UN EXEMPLE : LA NOTION DE TRANSITIVITÉ}

Comme on l'a dit plus haut, la notion de transitivité est exprimée dans les deux traditions par des termes équivalents, diábasis ou metábasis en grec, $a^{\varsigma} a d d \bar{\imath}$ en arabe, reposant sur la même métaphore, " traverser, franchir une limite»; en outre, leur usage métalinguistique n'est sûrement pas antérieur à la grammatisation, contrairement à des termes comme harf ('lettre') ou kalām ('discours, énoncé') mentionnés plus haut. On serait donc tenté de conclure sans autre forme de procès qu'il s'agit d'un emprunt de l'arabe au grec. Les choses, toutefois, ne sont pas si simples.

Tout d'abord se pose un problème de transmission. La notion de transitivité, en effet, n'apparaît pas dans la Technè de Denys le Thrace et n'a été introduite dans la grammaire syriaque qu'au XIII ${ }^{\mathrm{e}}$ siècle sous l'influence de la tradition arabe (Bohas 2020). Il faut donc supposer qu'elle serait passée directement du grec à l'arabe en court-circuitant l'intermédiaire habituel. La chose n'est évidemment pas strictement impossible, mais elle ne laisse pas d'être curieuse.

Ensuite, il y a un problème d'interprétation: que signifie au juste cette métaphore? Les grammairiens de part et d'autre lui donnent-ils le même sens ? Pour Apollonius Dyscole, les choses sont claires : il s'agit de «transitivité des personnes " (metábasis tōn prosōpōn), "personne » étant à entendre au sens d'« actant» (Lallot 1997 : I, 197-198 et n. 234). Autrement dit, c'est l'action exprimée par le verbe qui «transite» de l'agent au patient. Qu'en est-il maintenant chez Sībawayhi ? Comme l'a établi Aryeh Levin (1979), la transitivité est chez lui inséparable de la notion de rection ('amal); dans ce cadre, la métaphore

18 Comme c'est le cas, par exemple, de la Muqaddima fì l-naḥw attribuée à Halaf al-Aḥmar (mort en 796); il existe plusieurs autres ouvrages de ce type, datant du $\mathrm{IX}^{\mathrm{e}}$ et du début du $\mathrm{X}^{\mathrm{e}}$ siècle qui se caractérisent par des dimensions réduites et un niveau de théorisation des plus rudimentaires. 
prend un sens tout différent : le verbe, après avoir « agi » (c'est le sens exact de 'amila, que l'on traduit habituellement par 'régir') sur le sujet en lui assignant la marque du nominatif, « traverse » ce dernier pour assigner l'accusatif à l'objet ${ }^{19}$. Au demeurant, comme le souligne Sībawayhi, le complément d'objet n'est pas le seul dans ce cas :

Sache que le verbe qui ne traverse pas son agent [i.e. le verbe intransitif] traverse vers le nom de l'événement dont il [le verbe] est dérivé [...]. C'est [comme] lorsque tu dis qad dahaba 'Abdullāhi al-d̄ahāba al-šadīda ['Abdallāh-NOM s'est éloigné l'éloignement-ACC vif-ACC, i.e. " 'Abdallāh s'est éloigné vivement»]. (Kitāb : I, 11)

Cette construction, très fréquente en arabe classique, correspond à un complément d'objet interne; elle sert notamment à qualifier le procès. Mais l'important est qu'elle montre clairement que la transitivité telle que la conçoit Sībawayhi est une propriété du verbe en tant que catégorie formelle, indépendamment de la nature du procès qu'il signifie et des actants qu'il implique.

On pourrait dès lors être tenté de conclure que ces deux conceptions de la transitivité, en dépit des apparences, n'ont en fait rien de commun à part le nom, ce qui, s'ajoutant aux problèmes de transmission évoqués plus haut, invaliderait la thèse de l'emprunt, ou du moins l'affaiblirait considérablement. Toutefois, la tradition arabe a conservé les traces d'une conception plus ancienne, attribuée aux grammairiens de Kūfa et mise au jour par Djamel Kouloughli (2016 [1992]) : selon ces grammairiens, seuls seraient transitifs ( muta $^{\varsigma} a d d \bar{l}$, « franchissants ») les verbes exprimant une action qui «sort» réellement du sujet en direction de l'objet, que ce soit pour affecter celui-ci (e.g. daraba, 'frapper') ou pour le produire (e.g. bana, 'construire, édifier') ; en revanche, ils considèrent comme intransitifs (lāzim, 'inhérents') les verbes signifiant un processus affectant le sujet, et dont l'objet représente la source (e.g. hašiya, 'craindre'; 'ašiqa, 'être épris' avec accusatif de la personne; sami ${ }^{\varsigma} a$, 'entendre' $)^{20}$. Nous retrouvons bien ici la même conception " matérialiste " - si l'on peut dire - de la transitivité que chez Apollonius. Bref, il s'agirait d'une réalisation typique du scénario proposé par Talmon: la conception originelle, empruntée telle quelle par les premiers grammairiens arabes à la grammaire grecque, aurait ensuite été supplantée par celle de Sỉbawayhi, tout en se maintenant quelque temps chez les grammairiens de Kūfa.

Mais parvenus à ce point, nous nous heurtons à une nouvelle difficulté, d'ordre cette fois terminologique. En effet, nous ne connaissons l'interprétation kūfienne que par une source tardive ${ }^{21}$, qui reformule habituellement la doctrine kūfienne dans la terminologie, d'origine bașrienne, devenue canonique à l'époque. Or les

19 Rappelons que l'arabe est une langue VSO.

20 Il est intéressant - bien qu'un peu hors sujet - de noter que ces verbes, en arabe, appartiennent typiquement à une classe morphologique spécifique, la classe fa'ila; or Jean Lallot me signale qu'ils correspondent à peu près, en grec, à une classe particulière de verbes, identifiée par Apollonius Dyscole, qui prennent un complément au génitif et non à l'accusatif (voir Lallot 1997 : I, 262-265).

21 Il s'agit du Kitāb al-Inșāf d'al-'Anbārī (mort en 1181), un ouvrage consacré aux questions controversées entre les grammairiens de Bașra et de Kūfa. 
sources kūfiennes authentiques dont nous disposons n'utilisent pas $\operatorname{ta}^{\varsigma} a d d \bar{a}$ ('franchir') pour exprimer la transitivité, mais waqa'a 'ala ('tomber sur, porter sur' $\left.^{\prime}\right)^{22}$ : nous retrouvons bien la conception « matérialiste » de la transitivité, mais pas la métaphore du franchissement commune à Apollonius et à Sībawayhi.

Rien de tout cela, assurément, ne permet d'invalider définitivement la thèse de l'emprunt, qui conserve en l'occurrence une certaine plausibilité. C'est néanmoins une belle illustration, à propos d'un cas qui paraissait à première vue totalement limpide, des difficultés que rencontre l'hypothèse grecque dès qu'elle sort du domaine des généralités : confronté à des données lacunaires, ambiguës et contradictoires, le chercheur peut tout au plus parvenir à une intime conviction, avec ce que cela implique d'individuel et de subjectif, mais pas au genre de certitude sur laquelle on peut fonder un consensus.

\section{CONCLUSION}

Il faut donc nous y résigner : les origines de la tradition grammaticale arabe restent en grande partie obscures. Les plus anciens textes dont nous disposions ont été écrits par des savants qui appartiennent à peu près à la même génération, celle de Sïbawayhi ${ }^{23}$ et témoignent d'une réflexion grammaticale en plein essor ${ }^{24}$, capable de discuter de questions hautement sophistiquées, même si les grammairiens ne disposent que d'un métalangage encore fruste et d'une terminologie technique faiblement stabilisée. Ces discussions mettent en jeu des outils de provenances diverses : certains sont peut-être issus de la tradition gréco-syriaque, mais le recyclage qu'ils ont subi les a rendus presque méconnaissables; d'autres, et non des moindres, ont été fabriqués, si l'on peut dire, sur place ${ }^{25}$; d'autres enfin ont pu être élaborés en commun avec les premiers théoriciens du droit.

Qu'en est-il, dans ces conditions, de l'hypothèse grecque ? Comme on l'a dit plus haut, elle fournit un scénario plausible : le rejeter amènerait à supposer que la grammaire arabe s'est constituée ex nihilo, ou qu'elle a jailli tout armée du cerveau - si génial soit-il - de Sībawayhi, ce qui n'est guère satisfaisant. Mais elle ne fournit que cela : si loin que nous remontions, la tradition grammaticale a déjà pris une orientation totalement nouvelle et fabriqué, ou recyclé, ses outils en conformité avec les objectifs spécifiques qu'elle s'est fixés. Bref, on l'aura compris, la grammaire arabe n'est pas de la grammaire grecque étendue.

22 Voir par exemple al-Farrā', $\operatorname{Ma}^{\varsigma} \bar{a} n \bar{l} l-Q u r^{\top} \bar{a} n$ : II, 150, ainsi que Kinberg $1996: 969$.

23 Sībawayhi meurt, relativement jeune, vers 796, al-Farrā' et Abū 'Ubayda en 822, et Abū 1-Hasan alAhfaš en 830 : on peut supposer qu'ils sont tous nés entre la fin des années 740 et le début des années 760 . Les deux derniers sont auteurs de commentaires grammaticaux du Coran, comme al-Farrā?

24 Voir l'intéressant témoignage du Catholicos Timothée I ${ }^{\mathrm{er}}$, dans King 2012 : 199-201.

25 Outre la notion de rection ('amal) qui n'existe ni chez Denys ni chez Apollonius (Lallot 1997 : I, 288, n. 39), on peut citer la notion de « schème » (wazn) en morphologie, et la technique consistant à représenter ce dernier en remplaçant les trois consonnes radicales par celles du verbe fa'ala (« faire »). 


\section{RÉFÉRENCES}

?Anbārī (al-), Abū 1-Barakāt. Kitāb al-Inșāff, éd. établie par Muḥammad Muḥȳi 1-Dīn 'Abd al-Hamīd. 2 vol. Beyrouth : Dār al-Jīl. 1982.

Baalbaki, Ramzi. 2005. Rafael Talmon, Eighth-Century Iraqi Grammar: A Critical Exploration of Pre-Halīlian Arabic Linguistics (Harvard Semitic Studies 53). Eisenbrauns, Winona Lake 2003. Pp. xiv + 347. Price: $\$ 44.95$ hardback. ISBN: 1 57506-912-1. Journal of Semitic Studies 50 : 413-416.

— 2008. The Legacy of the Kitāb: Sībawayhi's Analytical Methods within the Context of the Arabic Grammatical Theory. Leyde \& Boston : Brill.

Bernards, Monique. 1997. Changing Traditions: Al-Mubarrad's Refutation of Sïbawayhi and the Subsequent Reception of the Kitāb. Leyde, New York \& Cologne : Brill.

Blachère, Régis. 1952-1966. Histoire de la littérature arabe, vol. I. Paris : Librairie d'Amérique et d'Orient Adrien-Maisonneuve.

Bohas, Georges. 2020. Transitivité et intransitivité dans la grammaire de Bar Hebraeus. HEL 42(1) : 49-57.

Carter, Michael G. 1972. Les origines de la grammaire arabe. Revue des études islamiques 40 : 69-97.

- 1973. An Arab Grammarian of the Eighth Century AD. A Contribution to the History of Linguistics. Journal of the American Oriental Society 93.2 : 146-157.

- 1985. When Did the Arabic Term Nahw First Come to Denote Grammar? Language and Communication 5 : 265-272.

- 2004. Sibawayhi. Londres \& New York : Tauris.

- 2016. Sībawayhi's Principles: Arabic Grammar and Law in Early Islamic Thought. Atlanta : Lockwood Press.

Elamrani-Jamal, Abdelali. 1983. Logique aristotélicienne et grammaire arabe (Études musulmanes XXVI). Paris : Librairie philosophique J. Vrin.

- 1984. C.H.M. Versteegh, Greek Elements in Arabic Linguistic Thinking, Leyde, E.J. Brill, 1977, 243 p., in- $8^{\circ}$ avec index et un ensemble de citations de textes originaux grecs et arables. Bulletin critique des Annales islamologiques I : 304306.

Farrā̄ (al-), Abū Zakariyā Yahyā. Ma ānī l-Qur'ān. $3^{\mathrm{e}}$ éd. 3 vol. Beyrouth : ${ }^{\complement} \bar{A} l a m$ alKutub. 1983.

Guillaume, Jean-Patrick. 2011. Defining the Word within the Arabic Grammatical Tradition: Astarābādī's Predicament. The Word in Arabic, dir. par Giuliano Lancioni et Lidia Bettini, 49-68. Leyde \& Boston : Brill.

- 2017. Phrase (jumla) et énoncé (kalām) dans la tradition grammaticale arabe. Langages 205 : 59-72.

- 2019. Les parties du discours dans la tradition grammaticale arabe. Histoire des parties du discours (Orbis Supplementa 46), dir. par Bernard Colombat et Aimée Lahaussois, 475-490. Louvain : Peeters.

Hugonnard-Roche, Henri. 2013. Sur la lecture tardo-antique du Peri hermeneias d'Aristote : Paul le Perse et la tradition d'Ammonius. Studia graeco-arabica 3 : 37-103. 
Humbert, Geneviève. 1995. Les voies de la transmission du Kitāb de Sïbawayhi. Leyde, New York \& Cologne : Brill.

Ibn al-Muqaffa ${ }^{\varsigma}$, Abdallāh. Al-manțiq li-Ibn al-Muqaffa ${ }^{\varsigma}$ suivi de Hudūd al-manțiq liIbn Bițrīq, éd. établie par Muḥammad Taqī Dāneš Pažūh. Téhéran : s.n. 1978.

Ibn al-Nadīm. Kitāb al-Fihrist, éd. établie par Gustav Flügel. 2 vol. Leipzig: F. C. W. Vogel. 1872.

Ildefonse, Frédérique \& Jean Lallot. 2017. Grec logos : premières approches philosophiques et grammaticales de l'énoncé. Langages 205 : 73-86.

Kinberg, Naphtali. 1996. A Lexicon of Farrä's's Terminology in his Qur'ān Commentary. Leyde, Boston \& Cologne : Brill.

King, Daniel. 2012. Elements of the Syriac Grammatical Tradition as These Relate to the Origins of Arabic Grammar. The Foundations of Arabic Linguistics: Sïbawayhi and Early Arabic Grammatical Theory, dir. par Amal Elesha Marogy, 189-209. Leyde \& Boston : Brill.

- 2013. Grammar and Logic in Syriac (and Arabic). Journal of Semitic Studies LVIII/1 : 101-120.

Kouloughli, Djamel Eddine. 2016 [1992]. Sur les notions de transitivité et d'intransitivité : considérations théoriques à propos d'une question controversée entre grammairiens de Bașra et de Kūfa. Trad. par Jean-Patrick Guillaume. Bulletin d'études orientales 65 : 13-26 (publication originale : Fī mafhūmay altåaddī wa-l-luzūm : ta’ammulāt naẓariyya ḥawla mas’alatin hilāfiyya bayna alnaḥwiyyīn al-bașriyyīn wa-l-kūfiyyīn. Al-Tawāṣul al-lisānī 4(2) : 41-52).

Lallot, Jean. 1997. Apollonius Dyscole. De la construction. 2 vol. Paris : Librairie philosophique J.-Vrin.

Levin, Aryeh. 1979. The Meaning of Ta $a^{\varsigma} d d \bar{a} A l-f i^{\varsigma} l$ 'ilā in Sībawayhi's Al-Kitāb. Studia Orientalia Memoriae D. H. Baneth Dedicata, dir. par Joshua Blau, Shlomo Pines, Meir Jacob Kister, Shaul Shaked, 193-210. Jérusalem : Magnes Press.

- 1981. The Grammatical Terms Al-Musnad, Al-Musnadi 'Ilayhi and Al-'Isnād. Journal of the American Oriental Society 101 : 145-165.

Merx, Adalbert. 1889. Historia artis grammaticae apud Syros (Abhandlungen für die Kunde des Morgenlandes IX.2). Leipzig : F. A. Brockhaus.

Rundgren, Frithiof. 1976. Über den griechischen Einfluss auf die arabische Nationalgrammatik. Acta Universitatis Upsaliensis n. s. 2(5) : 119-144.

Sībawayhi. Kitāb Sībawayhi, éd. établie par Hartwig Derenbourg. 2 vol. Paris, Imprimerie nationale. 1881-1889 (réimpr. Hildesheim \& New York: GeorgOlms-Verlag. 1970).

Talmon, Rafael. 1982. Naḩwiyyūn in Sībawayhi's Kitāb. Zeitschrift für arabische Linguistik $8: 12-38$.

- 1987. Musnad, Musnad Ilayhi and the Early History of Arabic Grammar: A Reconsideration. Journal of the Royal Asiatic Society 1987(2) : 208-222.

- 1990. The Philosophizing Farrầ?: An Interpretation of an Obscure Saying Attributed to the Grammarian Ta $a^{\complement}$ lab. Proceedings of the Second Workshop on the History of Arabic Grammar (Studies in the History of the Language Sciences 56), dir. par C. H. M. Versteegh \& Michael G. Carter, 265-279. Amsterdam \& Philadelphie : John Benjamin's. 
- 1993. The Term Qalb and its Significance for the Study of the History of the Early Arabic Grammar. Zeitschrift für Geschichte der Arabisch-Islamischen Wissenschaften $8: 71-113$.

- 2000. The First Beginnings of Arabic Linguistics: The Era of the Old Iraqi School. History of the Language Sciences, dir. par Sylvain Auroux, E. F. K. Koerner, Hans-Josef Niederehe \& Kees Versteegh (Handbücher zur Sprach- und Kommunikationswissenschaft 18.1), vol. I, 245-252. Berlin \& New York : Walter de Gruyter.

- 2003. Eighth-Century Arabic Grammar: A Critical Exploration of Pre-Halïlian Arabic Linguistics (Harvard Semitic Studies 53). Winona Lake : Eisenbraun's.

- 2005. Ġāya, Sifa and Al-Kalām Al-Wāṣif in Ibn al-Muqaffa's Manual of Logic: Some New Considerations about the Beginning of Arabic Grammar. Jerusalem Studies in Arabic and Islam 30 : 506-520.

Teixidor, Javier. 2003. Aristote en syriaque: Paul le Perse, logicien du ve siècle. Paris : CNRS Éditions.

Troupeau, Gérard. 1976. Lexique-index du Kitāb de Sībawayhi. Paris : Klincksieck.

- 1981. La logique d'Ibn al-Muqaffa' et les origines de la grammaire arabe. Arabica XXVIII : 242-250.

Versteegh, Kees. 1977. Greek Elements in Arabic Linguistic Thinking. Leyde : Brill.

- 1993. Arabic Grammar and Qur'ānic Exegesis in Early Islam. Leyde, New York \& Cologne : Brill.

Vidro, Nadia. 2020. Grammars of Classical Arabic in Judaeo-Arabic. Intellectual History of the Islamicate World, 8(2-3) : 284-305 [https://brill.com/view/journals/ ihiw/8/2-3/article-p284_6.xml, consulté le 14/01/2021]. 